\title{
Hole dynamics in spin and orbital ordered vanadium perovskites
}

\author{
Sumio Ishihara \\ Department of Physics, Tohoku University, Sendai 980-8578, Japan
}

(November 7, 2018)

\begin{abstract}
Hole dynamics in spin and orbital ordered vanadates with perovskite structure is investigated. A mobile hole coupled to the spin excitation (magnon) in the spin G-type and orbital C-type (SG/OC) ordered phase, and that to the orbital excitation (orbiton) in the spin C-type and orbital G-type (SC/OG) one are formulated on an equal footing. The observed fragile character of the (SG/OC) order is attributed to the orbiton softening caused by a reduction of the staggered magnetic order parameter. It is proposed that the qualitatively different hole dynamics in the two spin-orbital ordered phases in vanadates can be probed by the optical spectra.
\end{abstract}

PACS numbers: 71.30.+h, 71.10.-w, 75.30.-m, 78.30.-j

One of the central issues in the correlated oxides is doped Mott insulator [1,2]. The prototypical compound is the high Tc superconducting cuprates (HTSC) where the parent Mott insulating state with the antiferromagnetic (AFM) long-range order is understood by the single-band electronic model with strong Coulomb interactions. Apart from the half filling, doped holes are strongly renormalized by interaction with spin excitations.

There is another class of Mott insulator where the orbital degeneracy of the transition-metal ion remains. Mobile carriers doped into this class of insulator couple to the spin and orbital arrays. Vanadates with the perovskite structure $R_{1-x} A_{x} \mathrm{VO}_{3}$ ( $R$ : a trivalent rare-earth ion, $A$ : a divalent alkaline-earth ion) are the likeliest candidate. A number of researches have been done in connection with the spin-orbital orders in insulating $R \mathrm{VO}_{3}$ [3-12]. In a $\mathrm{V}^{3+}$ ion, two electrons occupy the $t_{2 g}$ orbitals with the $S=1$ high-spin state. Two kinds of spinorbital orders are found in the ground state $[3,5]$ : the G-type (three-dimensional (3D) staggered) AFM order associated with the C-type (rod type) orbital one termed the (SG/OC) phase in $\mathrm{YVO}_{3}$, and the C-type AFM order with the G-type orbital one termed the (SC/OG) phase in $\mathrm{LaVO}_{3}$. Both the orders have the commonly occupied $d_{x y}$ orbital at each $\mathrm{V}$ site, and the alternately occupied $d_{y z} / d_{z x}$ orbitals with the AFM spin alignment in the $a b$ plane. A difference between the two is seen along the $c$ axis; the staggered spin (orbital) order associated with the uniform orbital (spin) one in the (SG/OC) ((SC/OG)) phase.

Apart from the well studied $R \mathrm{VO}_{3}$, little is known about nature of mobile holes in the spin-orbital ordered $R_{1-x} A_{x} \mathrm{VO}_{3}$. A dynamics of a $d_{x y}$ hole is only to be expected from the consequence in a well-known doped two-dimensional (2D) quantum AFM and is common in the (SG/OC) and (SC/OG) phases. A clear contrast is anticipated in a hole motion along the $c$ axis; in the (SG/OC) and (SC/OG) phases, a mobile hole is strongly coupled to the spin and orbital excitations, i.e. magnon and orbital, respectively. Orbiton, standing for a quan- tized object of the collective excitation in orbital order, is observed in the Raman scattering of $\mathrm{LaMnO}_{3}[13]$ and is also proposed in $R \mathrm{VO}_{3}$ [12]. Thus, the perovskite vanadates are idea for study of the quantum hole dynamics in a spin-orbital ordered states. One of the striking difference between the observed two phases is the stability; the (SG/OC) phase is rather fragile against the hole doping $[4,14]$. the $(\mathrm{SG} / \mathrm{OC})$ phase realized in $\mathrm{YVO}_{3}$ rapidly disappears by doping of holes about $x \leq 0.05$ in $\mathrm{Y}_{1-x} \mathrm{Ca}_{x} \mathrm{VO}_{3}$. This phase is changed into $(\mathrm{SC} / \mathrm{OG})$ which is stable, at least, up to $x \sim 0.4$. The (SC/OG) phase in a series of $\mathrm{La}_{1-x} \mathrm{Sr}_{x} \mathrm{VO}_{3}$ also survives until $x=0.178$ [5]. This fragile nature of $(\mathrm{SG} / \mathrm{OC})$ is not explained within the doped 3D AFM with $S=1$.

We present, in this letter, a theory of doped perovskite vanadates with spin-orbital orders. The hole dynamics associated with magnon and orbiton in the (SG/OC) and $(\mathrm{SC} / \mathrm{OG})$ phases are formulated based on the selfconsistent Born approximation (SCBA). The different quasi-particle (QP) properties between the two phases and the observed barely stable (SG/OC) phase are attributed to the softening of the orbital excitation.

Our starting point is the $t-J$ type model with the $t_{2 g}$ orbital degree of freedom, $\mathcal{H}=\mathcal{H}_{t}+\mathcal{H}_{J}+\mathcal{H}_{J T}$, derived from the generalized Hubbard model in the large on-site interaction limit [15]. The exchange term between nearest neighbor (NN) spins and orbitals has the form $\mathcal{H}_{J}=\mathcal{H}_{4} A_{2}+\mathcal{H}^{2} E+\mathcal{H}^{2} T_{1}+\mathcal{H}^{2} T_{2}$ where ${ }^{4} A_{2},{ }^{2} E,{ }^{2} T_{1}$, and ${ }^{2} T_{2}$ indicate the intermediate $\left(t_{2 g}\right)^{3}$ configurations of the exchange processes. The leading term involves the high-spin ${ }^{4} A_{2}$ state explicitly given as

$$
\mathcal{H}_{4} A_{2}=-\frac{J_{4} A_{2}}{6} \sum_{\langle i j\rangle}\left(2+\vec{S}_{i} \cdot \vec{S}_{j}\right)\left(A^{l}+B^{l}-2 C^{l}\right),
$$

where $\vec{S}_{i}$ is the spin operator with magnitude $S=1$. $J_{4} A_{2}\left(=t^{2} /\left(U^{\prime}-I\right) \equiv J\right)$ is the exchange interaction with the on-site inter-orbital Coulomb interaction $U^{\prime}$, the exchange interaction $I$ and the transfer intensity $t$ between $\mathrm{NN} \mathrm{V}$ ions. The other exchange interactions are expressed by $J$ and $r_{J}=I / U^{\prime}$. The orbital sector in $\mathcal{H}_{4} J_{2}$ involving $A^{l}, B^{l}$, and $C^{l}$, where $l(=x, y, z)$ indicates 
a direction of a bond, is expressed by the local orbital operators at sites $i$ and $j$. These describe the diagonal and off-diagonal components of the electric-quadrupole moments, $O_{i E \gamma}(\gamma=u, v)$ and $O_{i T_{2} \gamma}(\gamma=x, y, z)$, respectively, and the orbital angular moments $O_{i T_{1} \gamma}(\gamma=$ $x, y, z)$. There is no continuous symmetry in the orbital sector of $\mathcal{H}_{J}$. The $t$ term is given as

$$
\mathcal{H}_{t}=\sum_{\langle i j\rangle, \gamma, \sigma}\left(t_{i j}^{\gamma} c_{i \gamma \sigma}^{\dagger} c_{j \gamma \sigma}+\text { H.c. }\right) \text {. }
$$

The operator $c_{i \gamma \sigma}$ annihilates a $t_{2 g}$ electron at site $i$ with orbital $\gamma=(y x, z x, x y)$ and spin $\sigma=(\uparrow, \downarrow)$, and is defined in the Hilbert space where the $\left(t_{2 g}\right)^{3}$ configuration is excluded at each site. In the ideal perovskite crystal, the hopping between the different orbitals is prohibited, and one of $t_{i j}^{\gamma}$ 's is zero and other two are equal, e.g. $t_{i, i+\hat{z}}^{x y}=0$ and $t_{i, i+\hat{z}}^{y z}=t_{i, i+\hat{z}}^{z x} \equiv t$. In addition to the $t$ and $J$ terms, roles of the Jahn-Teller (JT) interaction described by $\mathcal{H}_{J T}=g \sum_{i \gamma} Q_{i E \gamma} O_{i E \gamma}$ are stressed in the insulating $R \mathrm{VO}_{3}$ [9]. $Q_{i E \gamma}$ 's $(\gamma=u, v)$ are the normal modes of an $\mathrm{O}_{6}$ octahedron with symmetry $E_{g} \gamma$ and are treated as classical valuables. The detail expression of the above Hamiltonian and studies of the orbital order/excitation are presented in Refs. [12] and [15]. Similar spin-orbital models for insulating $R \mathrm{VO}_{3}$ are derived by other authors $[7-10,16,17]$.

Dynamics of holes doped into the spin-orbital ordered vanadates are formulated by SCBA which has been applied to HTSC $[18,19]$ and the colossal magnetoresistive (CMR) manganites [20-22]. The electron operator is expressed in the slave-fermion representation in the large limit of $S$. We assume that the orbital excitation between the $d_{y z}$ and $d_{z x}$ orbitals couples effectively to the doped holes, since (i) the excitation between $d_{x y}$ and $d_{y z} / d_{z x}$ dose not propagate coherently (local modes) [12], and (ii) the $d_{x y}$ band is separated from the $d_{y z} / d_{z x}$ ones, i.e. the excitation energy between $d_{x y}$ and $d_{y z} / d_{z x}$ is higher [6]. The electron operator is rewritten as,

$$
c_{i \gamma \sigma}=\left[\delta_{\gamma=(x y)} \tilde{f}_{i} \tilde{s}_{i \sigma}^{\dagger}+\delta_{\gamma=(z x, y z)} f_{i} s_{i \sigma}^{\dagger} t_{i \gamma}^{\dagger}\right] P_{S} .
$$

We introduce two fermionic operators $\left\{\tilde{f}_{i}, f_{i}\right\}$ for charge, two bosonic operators $\left\{\tilde{s}_{i \sigma}, s_{i \sigma}\right\}$ for spin, and bosonic one $\left\{t_{i \gamma}\right\}$ for orbital with the local constraints $\tilde{f}_{i}^{\dagger} \tilde{f}_{i}+$ $\sum_{\sigma} \tilde{s}_{i \sigma}^{\dagger} \tilde{s}_{i \sigma}=1, f_{i}^{\dagger} f_{i}+\sum_{\sigma} s_{i \sigma}^{\dagger} s_{i \sigma}=1$ and $\sum_{\sigma} s_{i \sigma}^{\dagger} s_{i \sigma}=$ $\sum_{\gamma=(y z, z x)} t_{i \gamma}^{\dagger} t_{i \gamma}$. The operator $P_{S}$ projects onto the ${ }^{3} T_{1}$ high-spin state in the $\left(t_{2 g}\right)^{2}$ configuration. Then, $\mathcal{H}_{t}$ is decoupled into the $d_{y z} / d_{z x}$ orbital part and the $d_{x y}$ one as $\mathcal{H}_{t}=\mathcal{H}_{y z / z x}+\mathcal{H}_{x y}$ with $\mathcal{H}_{y z / z x}=\mathcal{H}^{(c)}+\mathcal{H}^{(a b)}$. The main term is $\mathcal{H}^{(c)}$ describing a motion of the $d_{y z} / d_{z x}$ hole along the $c$ axis of our present interest. In the large limit of $S$, we remain the down (up) boson operators in the up (down) spin/orbital sublattice. $\mathcal{H}^{(c)}$ has the form

$$
\mathcal{H}^{(c)}=\frac{4 t}{\sqrt{N}} \sum_{\vec{k}, \vec{k}^{\prime}} g\left(k_{z}\right) f_{\vec{k}} f_{\vec{k}^{\prime}}^{\dagger} u_{\vec{k}^{\prime}-\vec{k}}+H . c .,
$$

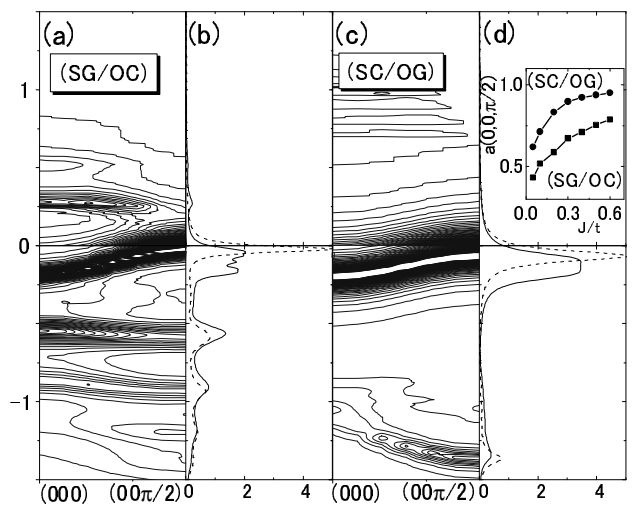

FIG. 1. Contour plot of spectral function and DOS of electron in (SG/OC) ((a) and (b)), and those (SC/OG) ((c) and $(\mathrm{d}))$ for $x=0.1$. Broken lines in (b) and (d) are for $A\left(\vec{k}=\left(0,0, \frac{\pi}{2 a}\right), \omega\right)$ multiplied by $1 / 5$. Parameters are $J / t=0.2, r_{J}=0.125$ and $g Q_{E} / J=1.25$. The origin of the vertical axis indicates the Fermi energy. The inset shows the $J$ dependence of the QP weight at $\vec{k}=\left(0,0, \frac{\pi}{2 a}\right)$.

where $u_{\vec{k}}=s_{\vec{k}}$ for the (SG/OC) phase and $u_{\vec{k}}=t_{\vec{k}}$ for (SC/OG) with $g\left(k_{l}\right)=\cos a k_{l}$. Explicitly, the mobile hole along the $c$ axis is coupled to magnon (orbiton) in the $(\mathrm{SG} / \mathrm{OC})((\mathrm{SC} / \mathrm{OG}))$ phase. $\mathcal{H}^{(a b)}$ and $\mathcal{H}_{x y}$, are common in the two phases. $\mathcal{H}^{(a b)}$ is for the $d_{y z} / d_{z x}$ hole in the $a b$ plane, and fomrs $f_{\vec{k}} f_{\vec{k}^{\prime}}^{\dagger} s_{\vec{q}}^{\dagger} t_{\vec{k}^{\prime}-\vec{k}+\vec{q}^{*}}^{\dagger}$ The simultaneous magnon-orbiton emissions/absorptions is expected to suppress the QP weight and dispersion. $\mathcal{H}_{x y}$ is for the $d_{x y}$ hole in the $a b$ plane, $\mathcal{H}_{x y}=\frac{4 t}{\sqrt{N}} \sum_{\vec{k}, \vec{k}^{\prime}}\left\{g\left(k_{x}\right)+\right.$ $\left.g\left(k_{y}\right)\right\} \tilde{f}_{\vec{k}} \tilde{f}_{\vec{k}^{\prime}}^{\dagger} \tilde{s}_{\vec{k}-\vec{k}^{\prime}}+$ H.c.. Similar physical consequences are only to be expected from this term to those in a mobile $d_{x^{2}-y^{2}}$ hole in the 2D quantum AFM studied in HTSC [18]. Here we focus on $\mathcal{H}^{(c)}$ characterizing different hole dynamics in the (SG/OC) and (SC/OG) phases. In the same scheme, $\mathcal{H}_{J}$ is formulated by the spin wave expansion [12]. In the magnon (orbiton) part of $\mathcal{H}_{J}$ written by $s_{i \sigma}\left(t_{i \gamma}\right)$, the orbital (spin) operators are replaced by the static correlation functions $\left\langle O_{i \Gamma \gamma} O_{j \Gamma^{\prime} \gamma^{\prime}}\right\rangle$ $\left(\left\langle\vec{S}_{i} \cdot \vec{S}_{j}\right\rangle\right)$, and the simultaneous spin-orbital excitations are neglected, same as those in $\mathcal{H}_{t}$ mentioned above. The lattice effects are limited to be static ones given by $\mathcal{H}_{J T}$, although the coupling with phonon may modify the QP band width and the orbiton dispersion around the crossing point with phonon in the small $J$ limit.

The electron spectral function $A(\vec{q}, \omega)$ is calculated from the fermion propagator $G(\vec{k}, \omega)$ where the boson operators are replaced by the saddle-point solutions. The self-energy for $G(\vec{k}, \omega)$ is given by

$$
\begin{aligned}
\Sigma(\vec{k}, \omega) & =\frac{i}{2 \pi N} \int d \nu \sum_{\vec{q}} G(\vec{k}-\vec{q}, \omega-\nu) \\
& \times\left|\widetilde{g}_{u}(\vec{k}, \vec{q})\right|^{2} D_{u 0}(\vec{q}, \nu),
\end{aligned}
$$

where $u=s$ for (SG/OC) and $u=t$ for (SC/OG). The 


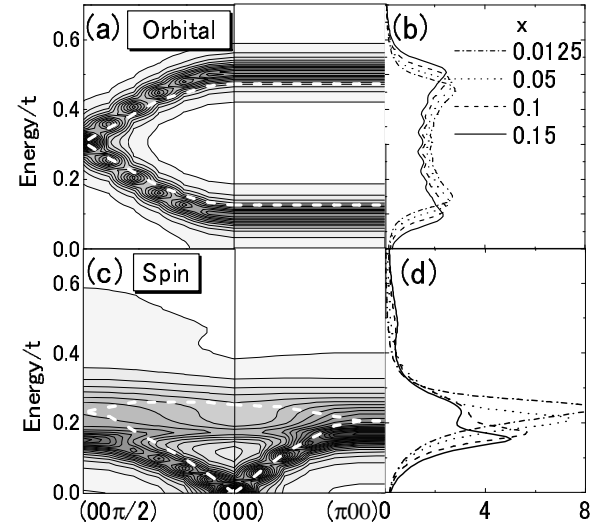

FIG. 2. Contour plots of the spectral functions at $x=0.1$ and the doping dependence of DOS for orbiton ((a) and (b)) and those for magnon ((c) and (d)) in (SG/OC). White broken lines in (a) and (c) indicate dispersions at $x=0$. The lower branch of the orbiton in (a) corresponds to that along $\left(0,0, \frac{\pi}{2 a}\right)-\left(0,0, \frac{\pi}{a}\right)-\left(\frac{\pi}{a}, 0, \frac{\pi}{a}\right)$ in the Brillouine zone for the cubic unit cell. Parameters are the same with those in Fig. 1.

bare magnon/orbiton propagators $D_{u 0}(\vec{q}, \nu)$ are used in Eq. (5) instead of the full propagators $D_{u}(\vec{q}, \nu) \cdot \widetilde{g}_{u}(\vec{k}, \vec{q})$ is the coupling constant including the coefficients in the Bogoliubov transformation. $A(\vec{k}, \omega)$ along $(0,0,0)-$ $\left(0,0, \frac{\pi}{2 a}\right)$, with $a$ being the cubic perovskite unit cell, and the density of states (DOS) $N(\omega)$ are obtained in the selfconsistent calculation (Fig. 1). A reasonable parameter set as $J / t=0.2, r_{J}=0.125$ and $g Q_{E} / J=1 \sim 1.25$ with $J$ being around $30 \mathrm{meV}$ is chosen based on the previous evaluations $[9,12]$. In both the two phases, $A(\vec{k}, \omega)$ shows the broad incoherent continuum and the well-separated QP peak near the Fermi level (FL). The dispersive QP peak has the band width $D$ of the order of $J$ and has the highest energy at the momentum $\left(0,0, \frac{\pi}{2 a}\right)$. The clear differences between the two phases are seen in the QP spectral weight $a(\vec{k})$ and $D$; in the (SC/OG) phase, a large portion of the spectra are concentrated on the QP peak with the larger width $D$, and $a(\vec{k})$ is rapidly saturated with increasing $J$ (the inset of Fig. 1). This is in contrast to the case of (SG/OC) where the spectra are dominated by the incoherent components. These are ascribed to the larger excitation energy of orbiton with the excitation gap about $2 J \sim 3 J$ (as shown later in Fig. 3(b)). We have checked and confirmed these features of $a(\vec{k})$ and $D$ by the variational-type calculations [23]. It is worth noting that the hole dynamics associated with the $t_{2 g}$ orbitons in (SC/OG) is in qualitatively contrast to that with the $e_{g}$ orbital for the CMR manganites [24,20]; $A(\vec{k}, \omega)$ has a large weight around the free $e_{g}$ bands rather than the QP part. This is because of the non-zero hopping matrices between the $\mathrm{NN}$ different $e_{g}$ orbitals unlike the $t_{2 g}$ ones.

Now we turn to the spin and orbital sectors. The renormalized magnon/orbiton propagators are given by utiliz-

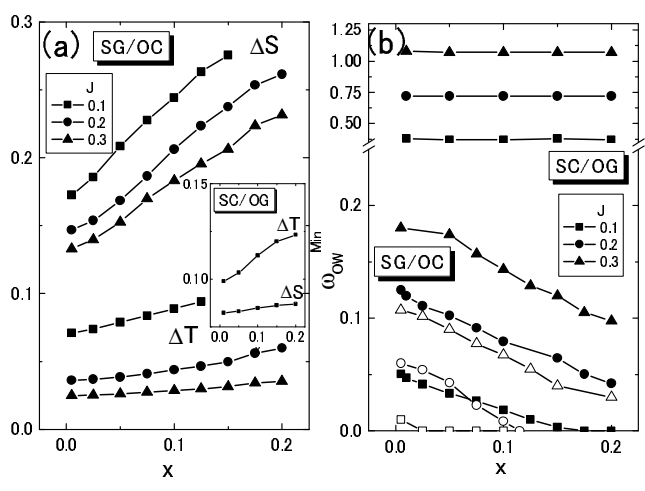

FIG. 3. (a) Doping dependences of reductions of the spin and orbital order parameters, $\Delta S$ and $\Delta T$, in (SG/OC). The inset shows those in $(\mathrm{SC} / \mathrm{OG}$ ) with $J / t=0.2$. (b) Doping dependence of the minimum orbiton energy $\omega_{O W}^{\mathrm{Min}}$. The filled and open symbols in (b) are for $g Q_{E} / J=1.25$ and 1 , respectively.

ing the calculated $G(\vec{k}, \omega)$. The bubble-type diagram is the lowest order of the self-energy,

$$
\begin{aligned}
\Pi_{u}(\vec{q}, \omega) & =\frac{-i}{2 \pi N} \int d \nu \sum_{\vec{k}}\left|\widetilde{g}_{u}^{t}(\vec{k}, \vec{q})\right|^{2} \\
& \times G(\vec{k}+\vec{q}, \omega+\nu) G(\vec{k}, \nu) .
\end{aligned}
$$

The spectral functions $S(\vec{q}, \omega)=(-1 / \pi) \operatorname{Im} D_{s}(\vec{q}, \omega)$ and $T(\vec{q}, \omega)=(-1 / \pi) \operatorname{Im} D_{t}(\vec{q}, \omega)$, and DOS in (SG/OC) are shown in Fig. 2. In the undoped case (white broken lines), the calculated $S(\vec{q}, \omega)$ well reproduces the experimentally observed spin wave dispersion in $\mathrm{YVO}_{3}$ [11]. The flat dispersion of $T(\vec{q}, \omega)$ in the $a b$ plane originates from no coherent propagation of the $d_{y z} / d_{z x}$ orbital excitations in this plane [12]. The excitation gap in $T(\vec{q}, \omega)$ at $x=0$ comes from the discontinuous symmetry of the orbital sector in $\mathcal{H}_{J}$ and the JT interaction, and is much smaller than that in (SC/OG). Namely, the fragile character is inherent in the C-type orbital order. As expected from distortions of the staggered spin/orbital array by mobile holes, softening and broadening are observed for $S(\vec{q}, \omega)$ in (SG/OC) (Figs. 2(c) and 2(d)) and for $T(\vec{q}, \omega)$ in (SC/OG) (not shown). Unexpectedly, the band width of $T(\vec{q}, \omega)$ in (SG/OC) becomes broader with doping (Figs. 2(a) and 2(b)), in spite that the orbital distortion due to the hole motion is not expected along the $c$ axis. The minimum energy $\omega_{O W}^{\mathrm{Min}}$ of $T(\vec{q}, \omega)$ in this phase is located at the point $\Gamma$ in the lowest branch which corresponds to the point $\left(0,0, \frac{\pi}{a}\right)$ in the original Brillouine zone for the cubic perovskite unit cell. $\omega_{O W}^{\mathrm{Min}}$ decreases with doping and finally touches the zero energy at small number of $x$. That is, the (SG/OC) phase is unstable against the small doping and is changed into the G-type orbital phase. This orbiton softening in (SG/OC) originates from reduction of the staggered spin order parameter, termed $\Delta S(>0)$ (see Fig. 3). The remarkable change by doping is only observed in $\Delta S$ of the (SG/OC) phase. 
However, this reduction itself is not sufficient for instability of the AFM ordering, i.e. $\Delta S<S(=1)$. Instead, the effective exchange interaction for the staggered (uniform) orbital alignment in $\mathcal{H}_{J}$, given as $J_{A F}^{o r b}=J_{4} A_{2}\left(2+\left\langle\vec{S}_{i} \cdot \vec{S}_{j}\right\rangle\right)$ $\left(J_{F}^{\text {orb }}=J_{\Gamma}\left(1-\left\langle\vec{S}_{i} \cdot \vec{S}_{j}\right\rangle\right)\left(\Gamma={ }^{2} E,{ }^{2} T_{1},{ }^{2} T_{2}\right)\right)$, rapidly increases (decreases) with increasing $\Delta S$. Finally, the orbiton energy becomes zero at the critical $x$ which depends on the exchange and JT interaction parameters. In short, the instability of the (SG/OC) phase with doping, observed in $\mathrm{Y}_{1-x} \mathrm{Ca}_{x} \mathrm{VO}_{3}$ [14], is caused by the orbiton softening due to increasing of $\Delta S$ through the spinorbital coupling.

The qualitatively different hole dynamics in the two phases can be probed by the optical experiments. The $z$-component of the optical conductivity spectra $\sigma_{z z}(\omega)$ is formulated in the slave-fermion scheme with the $1 / S$ expansion. The lowest order diagram of $\sigma_{z z}(\omega)$ is given by $\sigma_{z z}(\omega)=-\frac{(9 t e)^{2}}{c^{2}} \operatorname{Im} K(\omega)$ with

$$
\begin{aligned}
K(\omega) & =\frac{2}{(2 \pi N)^{2} \omega} \int d \omega_{1} d \omega_{2} \sum_{\vec{k}_{1}, \vec{k}_{2}}\left|\widetilde{g}_{u}\left(\vec{k}_{1}, \vec{k}_{2}\right)\right|^{2} G\left(\vec{k}_{1}, \omega_{1}\right) \\
& \times D_{u 0}\left(\vec{k}_{2}-\vec{k}_{1}, \omega-\omega_{1}+\omega_{2}\right) G\left(\vec{k}_{2}, \omega_{2}\right),
\end{aligned}
$$

for $u=s(u=t)$ in the (SG/OC) ((SC/OG)) phase, indicating the particle-hole pair creation associated with magnon/orbiton. Here we introduce the results of the regular part of $\sigma_{z z}(\omega)$ at finite frequency, rather than the Drude part (Fig. 4). With increasing holes, the sharp peak structure growing up around $\omega=J \sim 2 J$ in the (SC/OG) phase, reflecting the large QP weight in $A(\vec{k}, \omega)$ (Fig. 1), in contrast to the broad incoherent spectra in (SG/OC). A shoulder structure is clearly seen in the integrated spectral weight $M(\omega)=\frac{c^{2}}{(9 t e)^{2}} \int_{0}^{\omega} \sigma_{z z}\left(\omega^{\prime}\right) d \omega^{\prime}$ (the inset of Fig. 4) which would be an evidence characterizing the hole dynamics in the orbital ordered insulators.

In summary, we present a theory of doped vanadium perovskites with spin and $t_{2 g}$ orbital orders. The doped (SG/OC) and (SC/OG) phases are characterized by the hole motion along the $c$ axis scattered by magnon and orbiton, respectively. The QP dynamics associated with the $t_{2 g}$ orbital excitation show remarkable difference from that with magnon as well as the $e_{g}$ orbital excitation. Instability of the (SG/OC) phase into (SC/OG) observed in $\mathrm{Y}_{1-x} \mathrm{Ca}_{x} \mathrm{VO}_{3}$ is attributed to the orbiton softening through a reduction of the staggered spin order parameter. The angular resolved photoemission spectroscopy and optical experiments for the QP, as well as the inelastic neutron scattering for magnon and orbiton [12], are required to test directly the present theoretical results.

Author would like to thank T. Hatakeyama and S. Maekawa for their valuable discussions, and S. Miyasaka and Y. Tokura for providing their unpublished data. This work was supported by KAKENHI from MEXT, and KURATA foundation. Part of the nu-

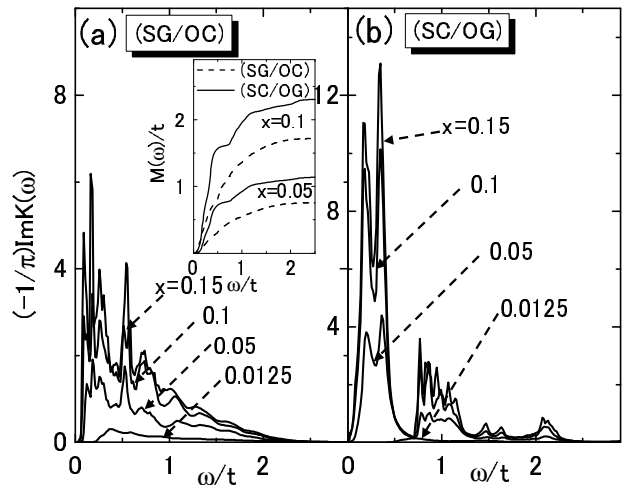

FIG. 4. Regular parts of the optical conductivity spectra for $(\mathrm{SG} / \mathrm{OC})(\mathrm{a})$, and those for (SC/OG) (b). The inset shows the integrated spectral weights for the (SG/OC) phase (broken lines) and those for the (SC/OG) ones (bold lines). Parameter values are the same with those in Fig. 1.

merical calculation has been performed by the supercomputers in IMR, Tohoku Univ., and ISSP, Univ. of Tokyo.

[1] M. Imada, et al., Rev. Mod. Phys. 70, 1039 (1998).

[2] S. Maekawa, et al., Physics of Transition Metal Oxides, (Springer Verlag, Berlin, 2004) and references therein.

[3] Y. Ren et al., Nature 396, 441 (1998).

[4] S. Miyasaka et al., Phys. Rev. Lett. 85, 5388 (2000).

[5] S. Miyasaka et al., Phys. Rev. B 68, 100406 (2003).

[6] H. Sawada et al., Phys. Rev. B 53, 12742 (1996).

[7] G. Khaliullin et al., Phys. Rev. Lett. 86, 3879 (2001).

[8] P. Horsch et al. Phys. Rev. Lett. 91, 257203 (2003).

[9] Y. Motome et al. Phys. Rev. Lett. 90, 146602 (2003).

[10] S.-Q. Shen et al. Phys. Rev. Lett. 88, 027201 (2002).

[11] C. Ulrich et al., Phys. Rev. Lett. 91, 257202 (2003).

[12] S. Ishihara, Phys. Rev. B 69, 075118 (2004).

[13] E. Saitoh et al., Nature, 410, 180 (2001).

[14] S. Miyasaka and Y. Tokura, (unpublished). In $\mathrm{YVO}_{3}$, the high temperature (SG/OC) phase is observed between $75 \mathrm{~K}$ and $120 \mathrm{~K}$, and the low temperature (SC/OG) one is below $75 \mathrm{~K}$. At around $x=0.02$ in $\mathrm{Y}_{1-x} \mathrm{Ca}_{x} \mathrm{VO}_{3}$, the (SC/OG) phase is changed into (SG/OC) which survives, at least, above $x=0.4$.

[15] S. Ishihara et al., Phys. Rev. B 65, 064442 (2002).

[16] K. I. Kugel et al., Sov. Phys. Solid State 17, 285 (1975).

[17] A. B. Harris et al., Phys. Rev. Lett. 91, 087206 (2003).

[18] C. L. Kane et al., Phys. Rev. B 39, 6880 (1989).

[19] G. Khaliullin et al., Phys. Rev. B 47, 463 (1993).

[20] J. van den Brink et al., Phys. Rev. Lett. 85, 5174 (2000).

[21] W. G. Yin et al., Phys. Rev. Lett. 87, 047204 (2001).

[22] J. Bala et al., Phys. Rev. Lett. 87, 067204 (2001).

[23] A part of the results is presented in S. Ishihara et al., Jour. Mag. Mag. Mat. 272-276, 412 (2004).

[24] W. Koshibae et al., Physica C 317-318, 205 (1999). 